\title{
Nonobtuse Triangulation of Polygons
}

\author{
Brenda S. Baker, Eric Grosse, and Conor S. Rafferty* \\ AT\&T Bell Laboratories, Murray Hill, NJ 07974, USA
}

\begin{abstract}
We show how to triangulate a polygon without using any obtuse triangles. Such triangulations can be used to discretize partial differential equations in a way that guarantees that the resulting matrix is Stieltjes, a desirable property both for computation and for theoretical analysis.

A simple divide-and-conquer approach would fail because adjacent subproblems cannot be solved independently, but this can be overcome by careful subdivision. Overlay a square grid on the polygon, preferably with the polygon vertices at grid points. Choose boundary cells so they can be triangulated without propagating irregular points to adjacent cells. The remaining interior is rectangular and easily triangulated. Small angles can also be avoided in these constructions.
\end{abstract}

\section{Introduction}

Can a polygon be triangulated without using any obtuse angles? This problem has been known for some time and solved manually in particular cases. For example, in an early paper [12] on discretizations of partial differential equations MacNeal says in an aside,

The network should be planar and none of the interior angles of the triangles should be obtuse. It may be necessary to insert a few additional points in order to fulfill the last condition.

A literature search (by looking for the keyword "triangulation" in on-line indices) and asking experts did not uncover any algorithms guaranteed to produce a

\footnotetext{
* Present address: Stanford University, ERL, Stanford CA 94305, USA.
} 
nonobtuse triangulation. Indeed, doubt was expressed as to whether such triangulations were even possible in general.

It turns out that elementary constructions suffice. Exactly how complicated the algorithm is depends on how many extra conditions are imposed dealing with small angles and interfaces, but no tools beyond high school geometry and trigonometry are needed.

To see why this problem is interesting, imagine solving a partial differential equation, say $\Delta u=f$, on a domain $P$. The finite element method chooses some approximating space $A$ and finds the function $u \in A$ such that for all $\nu \in A$, $\int_{P} \nu \Delta u=\int_{P} \nu f$. This leads to a matrix $\left[\int_{P} \nabla \phi_{i} \nabla \phi_{j}\right]_{i, j}$ involving basis elements $\phi \in A$. Frequently $A$ is composed of piecewise linear functions on a triangulation of $P$, with one degree of freedom at each triangle vertex. It is known [17, p. 78] that if there are no obtuse angles in the triangulation then for $i \neq j$ these integrals are negative and consequently the matrix is Stieltjes. Recall that a Stieltjes matrix is a symmetric positive definite matrix whose off-diagonal entries are all nonpositive. This property is important in the analysis of iterative methods for solving the linear system; for example, it implies that block Gauss-Seidel has better asymptotic rate of convergence than point Gauss-Seidel.

Discretizations such as the "box method" [18, p. 19] particularly benefit from nonobtuse triangulations. Consider Fig. 1.1, taken from [4]. The integrals mentioned above are replaced by quadrature rules in the following way. Form perpendicular bisectors to the triangle sides, drawn as dashed lines in the figure. Integrate $\Delta u$ over the dashed box and use the divergence theorem to express as an equivalent line integral, along the boundary, of the normal derivative of $u$. For each segment of the boundary, the normal derivative is estimated by a centered difference of function values at the two triangle vertices defining the bisector segment. It is desirable that bisectors corresponding to two sides of a triangle meet with that triangle; this will occur if and only if the triangle is not obtuse. Other schemes [13] have been devised that allow obtuse angles, but it is not clear whether they are as accurate.

The only algorithm for nonobtuse triangulation we are aware of is Bank's TRIGEN [3]. This routine uses heuristics with a single sweep across the polygon, starting with an initial distribution of points along the boundary. It is effective

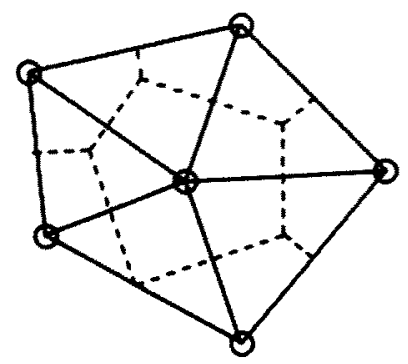

Fig. 1.1. A box formed by perpendicular bisectors. 
in practice but does sometimes fail. In such cases one starts again with a different initial distribution or corrects the triangulation manually.

Another feature of TRIGEN is that it tries to avoid small angles. It used to be thought that this was necessary for convergence of the finite element method, but [1] showed that it suffices to avoid large angles. Nevertheless, small angles may lead to ill-conditioned matrices [6], so it is best to avoid them.

If vertices are allowed on the sides of triangles, as in Fig. 2.1 below, then the existence of an acute triangulation is obvious and, moreover, a nearly equilateral triangulation is possible [8]. Unfortunately, continuity of linear elements is lost. Alternatively, if all the vertices of the triangles are preassigned, as in scattered data interpolation, then triangulation algorithms are available [5], [7], [9], [11], [16]. Several of these algorithms compute the Voronoi tessellation, which partitions the plane into polygonal regions by labeling an arbitrary point in the plane according to the closest vertex. Connecting vertices in adjacent regions gives the Delaunay triangulation. Actually the "no obtuse angles" is only a sufficient condition for the matrix to be Stieltjes. The necessary condition is that when two triangles adjoin in a side, the two angles opposite the side sum to at most $180^{\circ}$; the Delaunay triangulation achieves this weaker condition. But such compensation does not protect the integrals $\int \nu f$ when $f$ is nonlinear [10]. So we still seek a nonobtuse triangulation.

In this paper we give two solutions of increasing complexity. The first assumes that the vertices of $P$ lie on a square grid. The second removes this hypothesis and, moreover, avoids any angles smaller than $13^{\circ}$. Some details of the proof of the second method are omitted; see [2] (available from the authors).

\section{The Problem}

Given a simple polygon $P$ with vertices $\left\{\nu_{1}, \nu_{2}, \ldots, \nu_{n}\right\}$, add points $\left\{\nu_{n+1}, \ldots, \nu_{m}\right\}$ inside $P$ or on its boundary and connect the points with straight line segments to triangulate $P$. No resulting triangle should contain an obtuse angle. By a triangulation we mean a set of triangular regions such that the union is $P$; any two distinct triangles intersect along one full side, in a single point, or not at all; and the set of vertices of all the triangles is exactly $\left\{\nu_{i}\right\}_{1<1 \leq m}$. Figure 2.1 shows two triangulations that are illegal because of a point on a side and an obtuse angle, respectively, and Fig. 2.2 illustrates a legal triangulation.
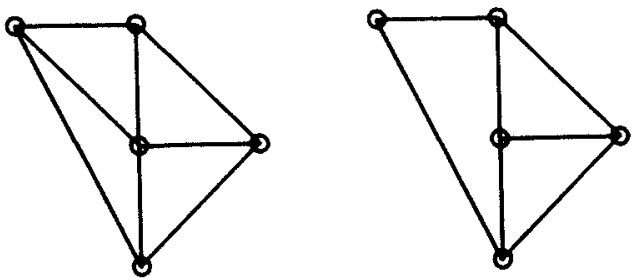

Fig. 2.1. Illegal triangulations. 


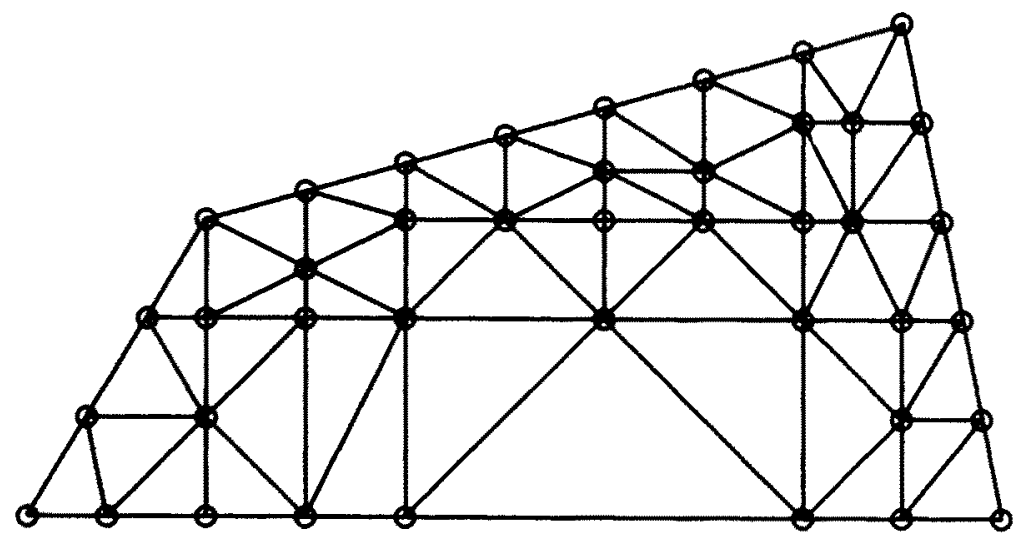

Fig. 2.2. A legal triangulation of a quadrilateral.

\section{Solution 1}

A natural approach is to partition the polygon. The trick is to divide in such a way that each subproblem can be solved independently and to prove for each subproblem that all cases have been considered.

Lemma 1. If the vertices of the polygon lie on a square grid and if none of the interior angles of the polygon are acute, then a nonobtuse triangulation exists.

Proof. Refine the grid until the cell diagonals are smaller than the minimum distance between nonintersecting boundary segments. Introduce points $\nu_{i}$ at the grid intersection points in the interior of $P$ and everywhere that a grid line intersects the boundary of $P$. Each square cell in the interior of $P$ is triangulated by adding a diagonal, leaving only cells intersecting the boundary to be dealt with. We will introduce some further points inside such cells and on the boundary of $P$, but not on the sides of the cells. Thus each cell is independently triangulated without propagating points from one cell to a neighbor.

If more than one boundary segment passes through a cell, the segments must be adjacent in order not to violate the refinement criterion. But they cannot have an acute interior angle. Therefore, they have an acute exterior angle, and the regions of $P$ bounded by these segments and the cell boundaries are disjoint. The triangulation strategy below can be applied independently to the two regions, each of which has only one boundary segment within the cell. We may assume without loss of generality that the upper right corner of the cell lies inside $P$ and the sides of the cell have length 1 . The boundary of $P$ will be indicated by a solid line and the cell by dashed lines.

Figure 3.1 illustrates two easy cases when the boundary hits the top and right or bottom sides. The bottom angle is acute because its vertex lies outside a semicircle drawn on the opposite side of the triangle.

When the left and bottom sides are hit, split the analysis into subcases based on the location of the point $q$ determined by vertical and horizontal lines extending 

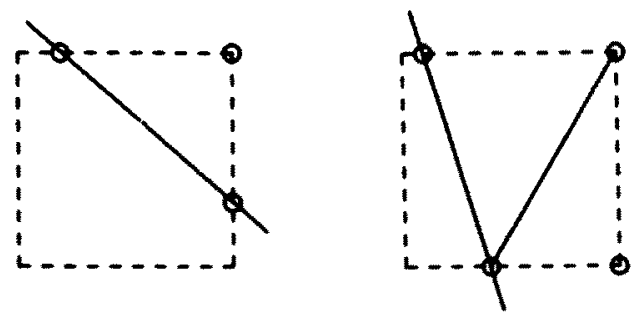

Fig. 3.1. Two easy cases.
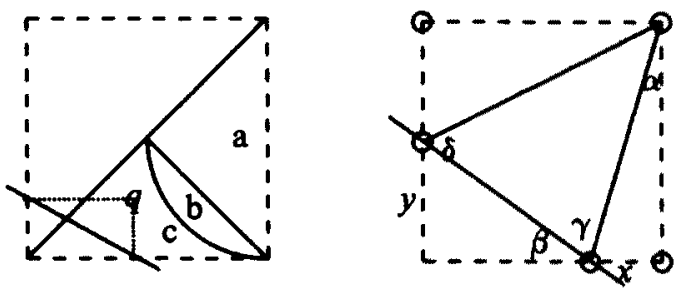

Fig. 3.2. Subcases.

from intersections of the boundary and the cell sides. Without loss of generality, we may assume that $q$ lies on or below the diagonal from the upper right corner to the lower left corner of the cell (see Fig. 3.2). Draw a semicircle based on the right side of the cell and another diagonal to form three regions $a, b$, and $c$ in which $q$ can lie.

In Subcase (a) we have $x \leq y$ and hence $\alpha \leq \beta$. So $\gamma=90^{\circ}+\alpha-\beta \leq 90^{\circ}$. To show $\delta \leq 90^{\circ}$, note that a semicircle based on the opposite side has radius at most $\sqrt{5} / 4$ and center at most $\frac{1}{4}$ away from the right side of the cell. In Subcase (b), $\gamma>90^{\circ}$. Introduce a point in the interior of the cell at the intersection of lines drawn to form five right triangles. $\delta<90^{\circ}$ because $y<\frac{1}{2}$. Similarly, in Subcase (c), the two nonright triangles are acute (Fig. 3.3).

Theorem 1. If the vertices of the polygon lie on a square grid, then there exists a nonobtuse triangulation.

Proof. For each vertex $\nu_{i}$ with an acute interior angle, cut off a corner by adding new vertices $\nu_{1}^{\prime}$ and $\nu_{i}^{\prime \prime}$ so that the triangle $\left\{\nu_{i}, \nu_{i}^{\prime}, \nu_{i}^{\prime \prime}\right\}$ being removed is acute and
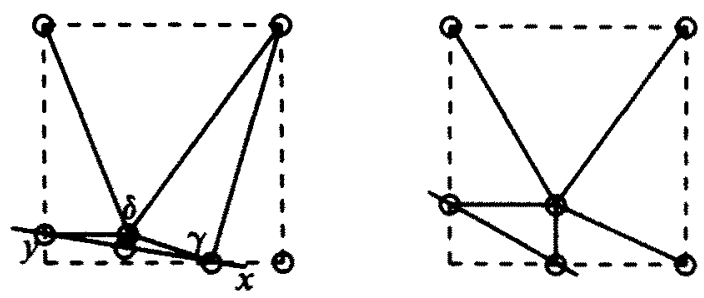

Fig. 3.3. Subcases (b) and (c). 

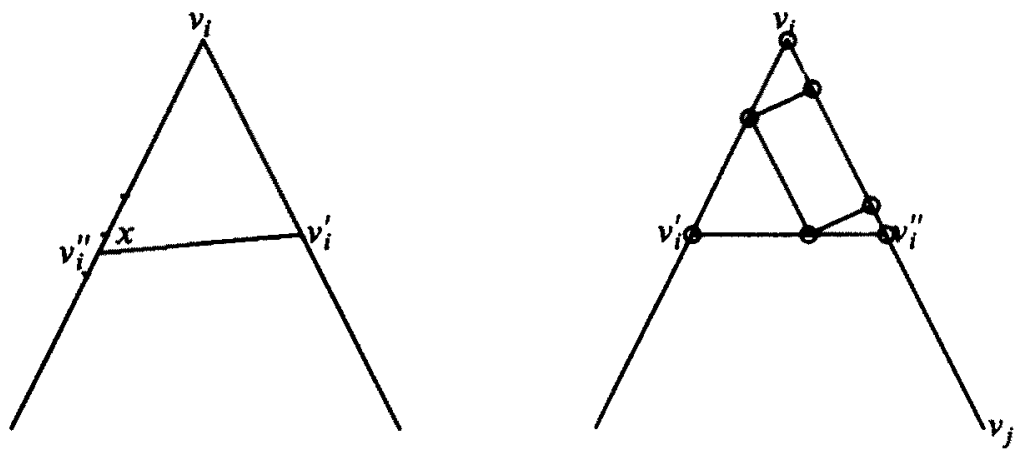

Fig. 3.4. Approximating an isosceles triangle on grid points.

does not contain any other vertices and so that the newly generated interior angles are obtuse. The only question is how to pick $\nu_{i}^{\prime}$ and $\nu_{,}^{\prime \prime}$. If they did not need to be on grid points, we could pick two points that form an isosceles triangle with $\nu_{i}$. We could thus cut off an acute triangle and leave obtuse interior angles, and by making the triangle small enough we could also guarantee that it does not contain other vertices. To obtain $\nu_{i}^{\prime}$ and $\nu_{i}^{\prime \prime}$ on grid points, we will approximate this soluion as follows. By hypothesis, the grid is fine enough so that there are grid points on the line between $\nu_{i}$ and each adjacent vertex such that the triangle formed by $\nu_{i}$ and these two grid points does not include any other vertices. If this triangle is satisfactory, let $\nu_{i}^{\prime}$ and $\nu_{i}^{\prime \prime}$ be its two new vertices. Otherwise, let $\nu_{i}^{\prime}$ be the new vertex that is closer to $\nu_{i}$ (see Fig. 3.4). The angle at $\nu_{i}^{\prime}$ within the current triangle must be obtuse, or the triangle would be satisfactory. Let $x$ be the point on the other side that is the same distance from $\nu_{i}$ as $\nu_{i}^{\prime}$. For some $\varepsilon$, picking any point within $\varepsilon$ of $x$ and within the current triangle will give a new acute triangle that does not contain any other vertices. Refining the grid sufficiently guarantees that a grid point will lie within this interval. Letting it be $\nu_{l}^{\prime \prime}$ gives a satisfactory triangle with vertices that are grid points.

Apply the lemma above to the new polygon, which does not have acute interior angles. This may introduce points on the artificial boundary segment. Figure 3.4 shows how to introduce orthogonal lines emanating from such points that partition the removed triangle into right triangles and rectangles, which of course can also be divided into right triangles.

The references to a "sufficiently fine grid" might suggest that many triangles are produced. But it is possible to refine the grid locally, as in Fig. 2.2. One way to do this is to use quadtrees [14], [15].

\section{Solution 2}

As mentioned earlier, it is desirable to avoid small angles. We have devised an algorithm that guarantees that no angle in the triangulation is less than $\tan ^{-1}\left(\frac{1}{3}\right) \approx$ $18^{\circ}$ or the minimum interior angle in the boundary, whichever is smaller. 
Moreover, if all interior angles of the polygon are at least $54^{\circ}$, then independent triangulations of abutting polygons are consistent, that is the same vertices will be created on the interface boundary segments. However, that solution still requires that the polygon vertices lie on a square grid.

So we move on to a more elaborate analysis which frees the vertices to lie in arbitrary position. Define a line to be nearly horizontal if its slope is at least -1 and at most 1 , and nearly vertical otherwise. A vertical grid line is nearly perpendicular to a nearly horizontal line; a horizontal grid line is nearly perpendicular to a nearly vertical line. Define a triangulation of a polygon to be good if it uses no obtuse angles and no angles less than $\tan ^{-1}\left(\frac{1}{4}\right)$ or the smallest angle in the polygon, whichever is smaller. A good triangulation well-triangulates the region.

Let $R$ be a simple polygon. The triangulation strategy will be to well-triangulate in the vicinity of each vertex of $R$, and then to well-triangulate the remaining region $R^{\prime}$. Figure 4.1 shows how $R$ might be divided into regions around each vertex and $R^{\prime}$. In triangulating the region around a vertex, points are introduced on the common boundary with $R^{\prime}$. No new points can be added on these common boundaries while triangulating $R^{\prime}$. (Points added on a common boundary would invalidate the triangulation already done in the adjoining region around the vertex.) The key to the proof is to restrict the edges occurring in the boundaries of the regions around the vertices so that the remaining region is easily welltriangulated.

Lemma 4.1. Let $R^{\prime}$ be a simple polygon overlaid with a unit grid. Suppose each edge of $R^{\prime}$ is of one of the following forms:

(1) cell diagonal,

(2) cell side,

(3) gridline segment forming the sides of two adjacent cells.

Then $R^{\prime}$ can be well-triangulated without adding any extra points on its boundary.

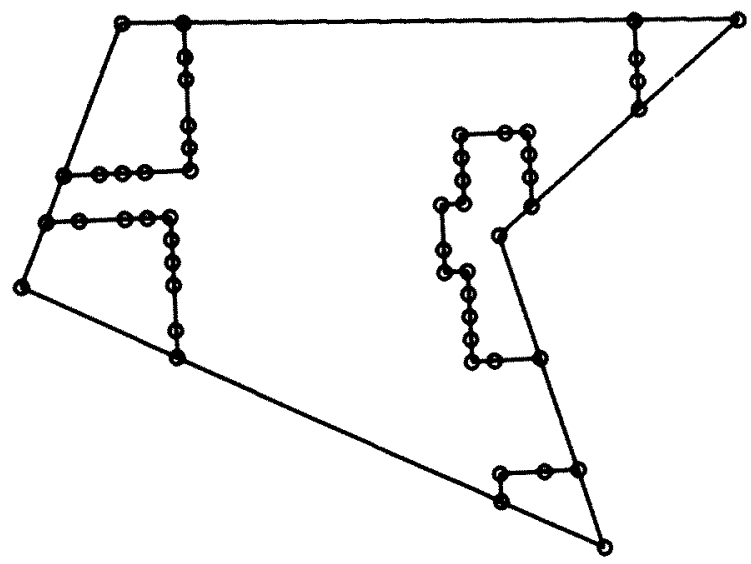

Fig. 4.1. Vertex cells. 


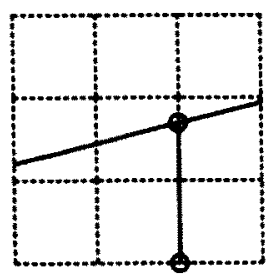

Fig. 4.2. A neighbor gridpoint.

Proof. Add a vertex at every interior gridpoint. Add edges on cell sides to connect previously unconnected vertices one unit apart. Add edges on cell diagonals where possible to do so without crossing or lying on top of another cell diagonal. Add a diagonal to each rectangle still lacking a diagonal. The proof that this procedure well-triangulates $R^{\prime}$ is by showing that every original edge borders a good triangle and every new edge borders a good triangle on each side.

If $e$ is a side of $R$, and $g$ is a gridline nearly perpendicular to $R$, intersecting $e$ at $A$, there is a unique gridpoint on $g$ whose distance from $A$ is at least one and less than two and which lies on the interior side of $e$. This gridpoint is called the neighbor gridpoint of $\boldsymbol{A}$. It is also called a neighbor gridpoint of $e$. Figure 4.2 illustrates a point on a side of $R$ and its neighbor gridpoint.

Let $e_{1}$ be a side of $R$, and let $A$ be an intersection point of $e_{1}$ with a nearly perpendicular gridline. Let $e_{2}$ be an adjacent side of $R$, and let $B$ be an intersection point of $e_{2}$ with a nearly perpendicular gridline. A sequence of edges from $A$ to $B$ is a satisfactory path from $A$ to $B$ if it lies in the interior of $R$ (except for $A$ and $B$ ), the edges are pairwise nonintersecting (except for the point between two successive edges), and each edge is of one of the following forms:

(1) a cell side or diagonal,

(2) a gridline segment forming the sides of two adjacent cells,

(3) $A G$, where $G$ is a neighbor gridpoint of $A$, or $B G$, where $G$ is a neighbor gridpoint of $B$.

Satisfactory paths are illustrated in Fig. 4.1. The region bounded by the path, $e_{1}$, and $e_{2}$ has a satisfactory boundary. An edge is satisfactory if it is of a form satisfying one of (1)-(3).

Lemma 4.2. Let $R$ be a simple polygon overlaid with a unit grid, such that no vertex of $R$ lies within four units of a nonadjacent edge. For each edge $e=(A, B)$, let $e_{A}$ and $e_{B}$ be points lying on gridlines nearly perpendicular to $e$, with $e_{A}$ closer than $e_{B}$ to $A$. Designate each gridpoint lying less than one unit from $e$ along a nearly perpendicular gridline between those of $A$ and $B$ as $a$ forbidden point. For each vertex $V$ with incident edges $e$ and $f$, let $P_{V}$ be a satisfactory path from $e_{V}$ to $f_{V}$, and let $R_{V}$ be the region bounded by $P_{v}, e_{v}$, and $e_{i}$. If these paths are pairwise nonintersecting and no path touches a forbidden point, then the region

$$
R-\bigcup_{V} R_{V}
$$

can be well-triangulated, with no new vertices introduced on any path $P_{\mathrm{V}}$. 


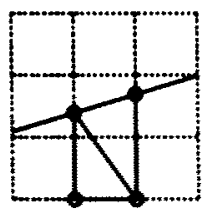

(a)

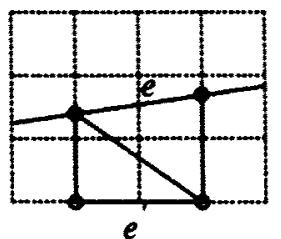

(c)

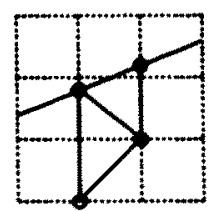

(b)

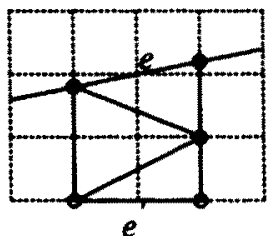

(d)

Fig. 4.3. Quadrilaterals along a nearly horizontal edge.

Proof. For each edge $e=(A, B)$, introduce a vertex on each nearly perpendicular gridline lying between $e_{A}$ and $e_{B}$. On each such gridline, also add a vertex at its neighbor gridpoint of $e$, unless that gridpoint is occupied by some path $P_{V}$.

Wherever two successive gridlines have vertices at neighbor points of $e$, connect them with an edge. This forms quadrilaterals with two parallel edges (see Fig. 4.3(a) and (b)). The side connecting the two gridpoints is either a horizontal or vertical edge of length one, or a cell diagonal. In either case, a diagonal of the quadrilateral well-triangulates it as desired. Since none of the satisfactory paths occupy either neighbor point or any forbidden points, and no vertex not adjacent to $e$ lies within four units of $e$, the new edges do not conflict with any previous edges.

Now, suppose a neighbor gridpoint of $e$ is occupied by a satisfactory path. If it does not lie on an edge of length 2 nearly parallel to $e$, it can form part of a quadrilateral formed as above with an adjacent neighbor gridpoint of $e$. If it lies in an edge $e^{\prime}$ of length 2 nearly parallel to $e$, then $e^{\prime}$ can t.rrm part of a well-triangulated quadrilateral as shown in Fig. 4.3(c) or (d), according to whether both endpoints of $e^{\prime}$ are neighbor gridpoints of $e$. Again, the new edges cannot conflict with any previous edges.

After triangulating along each original edge of $R$ as above, the region remaining to be triangulated can be well-triangulated by Lemma 4.1.

Thus, we need only show that we can triangulate around each vertex of $R$ so as to satisfy Lemma 4.2. By making the grid sufficiently fine, we will ensure that no two satisfactory paths intersect.

Vertices of $R$ need not be on grid points. For each vertex $A$, define the grid cell containing $A$ to be any grid cell for which $A$ is in the interior or on the 


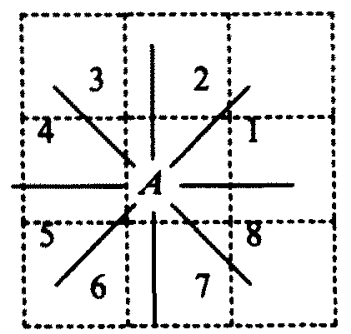

Fig. 4.4. Octants.

boundary. Number the octants at each vertex counterclockwise as shown in Fig. 4.4. Similarly, number the quadrants counterclockwise.

The next two lemmas will be helpful in triangulating around each vertex so as to obtain a satisfactory path along the boundary.

Lemma 4.3 Let $e$ be an edge of $R$ that is at an angle of $\rho$ to the vertical, $\rho<45^{\circ}$, as shown in Fig. 4.5(a)-(c), with the interior of $R$ to the left of e. Let $A$ be at the intersection of a horizontal gridline with e. Let $G$ be the gridpoint that lies at least 2 and less than 3 units to the left of $A$. Then one of the regions shown in Fig. 4.5 can be well-triangulated as shown, depending on the value of $A_{x}-G_{x}-2 \tan \rho$. The boundary from $G$ to $B$ is satisfactory for each region.

Proof. Let $d=A_{x}-G_{x}-2 \tan \rho$. The bounds on $\rho$ imply $d \in[0,3)$.

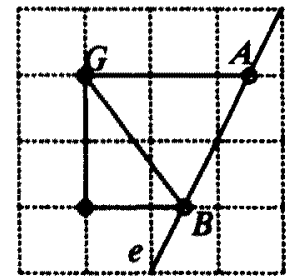

(a)

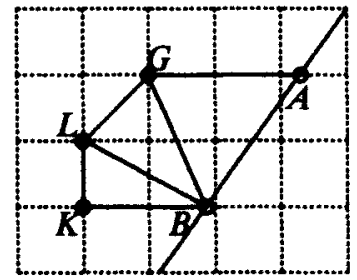

(b)

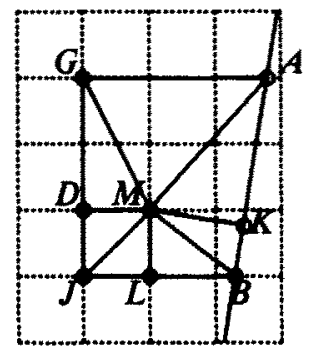

(c)

Fig. 4.5. Lemma 4.3. 
If $d \in[1,2)$, then triangulate as shown in Fig. 4.5(a). Since $B_{x}=G_{x}+d, \angle A G B$ is nonobtuse; since $G_{y}-B_{y}=2, \angle A G B \geq 45^{\circ}$. Angle $G A B=\pi / 2-\rho$ is between $45^{\circ}$ and $90^{\circ} . \angle G B A$ is non-obtuse because $|G A|$ is less than twice the height of the triangle, and at least $\tan ^{-1}\left(\frac{1}{2}\right)$ because dropping a perpendicular from $B$ to $G A$ gives at least one subangle with a tangent of at least $\frac{1}{2}$.

If $d \in[0,1)$, then triangulate as shown in Fig. 4.5(b). Let $\theta$ be the angle of $G B$ with the vertical. Since $d<1, \theta \leq \tan ^{-1}\left(\frac{1}{2}\right)$. Since $\angle L B K \leq 45^{\circ}$ and $\theta \leq \tan ^{-1}\left(\frac{1}{2}\right)$, $\angle G B L \geq 45^{\circ}-\tan ^{-1}\left(\frac{1}{2}\right)>18^{\circ}$. Since $\angle G L B=135^{\circ}-\angle K L B$ and $K L B \in$ $\left[45^{\circ}, \tan ^{-1}(2)\right], \angle G L B \in\left[45^{\circ}, 90^{\circ}\right]$.

Finally, suppose $d \in[2,3)$. Then $\rho<\tan ^{-1}\left(\frac{1}{2}\right)$. Let $d^{\prime}=d-\tan \rho$. Then $d^{\prime} \in$ $[1.5,3)$. If $d^{\prime} \in[1.5,2)$, triangulate as shown in Fig. $4.5(\mathrm{c})$, without the vertex $L$ and the edge $M L$. If $d^{\prime} \in[2,3)$, include $L$ and the edge $M L$. The edge $M K$ is drawn perpendicular to $A B$. Since $\rho<\tan ^{-1}\left(\frac{1}{2}\right),|A K| \leq \sqrt{5}$. Also, $|M K| \geq 1$ because $d \geq 2$. Therefore, $\angle M A K \geq \tan ^{-1}(1 / \sqrt{5}) . \angle G A M$ is between $\angle A M K$ and 45. $\angle K B M=\rho+90^{\circ}-\angle M B J$. If $d^{\prime} \in[1.5,2), \angle M B J \in\left[45^{\circ}, \tan ^{-1}(2)\right]$, and $\angle K B M \in\left[\tan ^{-1}\left(\frac{1}{2}\right), 72^{\circ}\right]$. If $d^{\prime} \in[2,3)$, then $\rho \leq \tan ^{-1}\left(\frac{1}{3}\right)$ and $\angle M B J \in$ $\left[\tan ^{-1}\left(\frac{1}{2}\right), 45^{\circ}\right]$, implying that $\angle K B M \in\left[45^{\circ}, 82^{\circ}\right]$.

Lemma 4.4. Let $e$ be an edge of $R$ that is at an angle of $\rho$ to the vertical, $\rho \leq 45^{\circ}$, as shown in Fig. 4.6(a), with the interior of $R$ to the right of $e$. Let $A$ be at the intersection of a horizontal gridline with e. Let $G$ be a gridpoint that lies at least 2 and less than 4 units to the right of A. Then a quadrilateral AGHJ (as shown in Fig. 4.6(a)) can be triangulated, where $G H$ has length either 2 or 4, with vertices added on $\mathrm{GH}$ and $\mathrm{HJ}$ to make these sides satisfactory.

Proof. Let $x_{1}=|A G|$, and let $x_{2}=x_{1}+2 \tan \rho$. Then $x_{2} \in[2,6)$.

If $x_{2} \in[2,4)$, then triangulate as in Fig. 4.6(b), where $K$ is the neighbor vertex of $J$. Consequently, $|K H|$ is either 1 or 2 .

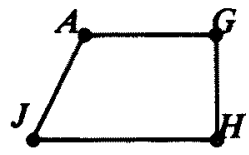

(a)

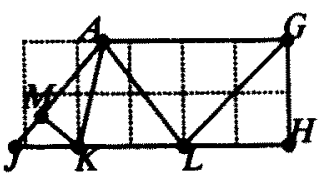

(c)

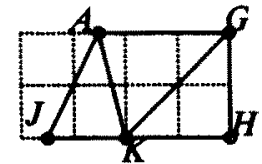

(b)

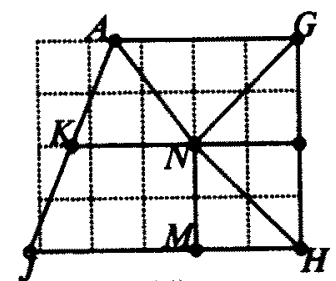

(d)

Fig. 4.6. Lemma 4.4. 
If $x_{1} \in[2,3)$ and $x_{2} \in[4,5)$, or if $x_{1} \in[3,4)$ and $x_{2} \in[5,6)$, triangulate as in Fig. $4.6(\mathrm{c})$, where $K$ is the neighbor vertex of $J, K M$ is perpendicular to $A J$, and $|L H|=2$. Consequently, $|K L|$ is either 1 or 2 . The relative sizes of $x_{1}$ and $x_{2}$ imply $\tan \rho \geq \frac{1}{2}$. Obviously, $\triangle L G H$ is good. The relative sizes of the height and bases of triangles $A K L$ and $A G L$ force those triangles to be good. $\angle M J K=90^{\circ}-\rho$. Since $|J K| \geq 1$ and $\angle M J K \geq 45^{\circ},|M K| \geq 1 / \sqrt{2}$. Note that the length of $A K$ is at most $\sqrt{5}$. Since $|M K| \geq 1 / \sqrt{2}$ and $|A K| \leq \sqrt{5}, \angle M A K \geq \tan ^{-1}(1 / \sqrt{10})>\tan ^{-1}\left(\frac{1}{4}\right)$. Also, $\angle M A K \leq \rho \leq 45^{\circ}$.

The only remaining case is when $x_{1} \in[3,4)$ and $x_{2} \in[4,5)$. Triangulate as in Fig. $4.6(\mathrm{~d})$, where $|K N| \in[2,3)$ and region $K N M J$ is to be filled in according to Fig. 4.6(b) or (c).

Lemma 4.5. Let $A$ be the vertex of an acute angle of $R$. Then there is a region around $A$ that can be well-triangulated with a satisfactory path along its boundary.

Proof. Let $\alpha$ be the angle at $A$. We may assume that one edge $L_{1}$ at $A$ lies in octant 6. The clockwise edge $L_{2}$ lies in one of octants 5-8. Let $\rho, 0 \leq \rho \leq 45^{\circ}$, be the angle of $L_{1}$ with the vertical.

Case 1. Suppose $L_{2}$ is in octant 5 . Let $\sigma, 0 \leq \sigma \leq 45^{\circ}$, be the angle of $L_{2}$ with the horizontal. For any positive constant $c$, there is a neighbor gridpoint $G$ of $L_{2}$ that also lies to the left of $L_{1}$ by a horizontal distance in the range $[c, c+1+\tan \rho)$. Form a parallelogram $P$ by lines parallel to $L_{1}$ at horizontal distances of $c$ and $c+1+\tan \rho$ from $L_{1}$ and by lines parallel to $L_{2}$ at vertical distances of 1 and 2 from $L_{2}$, as shown in Fig. 4.7(a). Then $V_{x}-U_{x} \geq 1$ and a vertical gridline lies between $V$ and $U$ or passes through $Q$. Since length 1 or this gridline lies in $P$, there is a gridpoint inside $P$ or on its right or upper boundary.

There are two subcases according to the values of $\rho$ and $\sigma$. First, suppose $\rho \leq \tan ^{-1}\left(\frac{1}{4}\right)$ and $\sigma \leq \tan ^{-1}\left(\frac{1}{4}\right)$. We begin by showing the existence of a gridpoint $H$ that is a neighbor gridpoint of both $L_{1}$ and $L_{2}$. Let $G$ be the neighbor gridpoint of $L_{2}$ found by the above argument with $c=1$, so that $G$ lies to the left of $L_{1}$ by a distance in $[1,2+\tan \rho)$. If the latter distance is less than 2 , we are done. Otherwise, move right one unit to a gridpoint $G_{1} . G_{1}$ lies to the left of $L_{1}$ by a distance in $[1,1+\tan \rho)$ and below $L_{2}$ by a distance in $[1+\tan \sigma, 2+\tan \sigma)$. If the latter distance is less than 2 , we are done. Otherwise, move up one unit to a gridpoint $G_{2} . G_{2}$ lies below $L_{1}$ by a distance in $[1,1+\tan \sigma)$ and to the left of $L_{2}$ by a distance in $[1+\tan \rho, 1+2 \tan \rho$ ).

Triangulate as shown in Fig. 4.7(b). Note that $\angle A Q R=90^{\circ}+\rho-\angle R Q H$ and $\angle R Q H \in\left[\tan ^{-1}\left(\frac{1}{2}\right), \tan ^{-1}(2)\right]$. So $A Q R$ and similarly $A R Q$ are good.

Now, suppose $\rho>\tan ^{-1}\left(\frac{1}{4}\right)$. From the earlier argument with $c=2$, there is a neighbor gridpoint $H$ of $L_{2}$ that lies to the left of $L_{1}$ by a distance in $[2,3+\tan \rho)$. Triangulate as shown in Fig. $4.7(\mathrm{c})$, where $R$ and $Q$ lie on the gridlines through $H, Q S$ is vertical, and $S T$ is perpendicular to $L_{1}$.

Unfortunately, $H$ is not the neighbor vertex of $Q$. Therefore, $R H Q$ is not a satisfactory path. If $|H Q| \in[2,3)$, apply Lemma 4.3. Otherwise, form a quadrilateral below $H Q$ by going down 2 from $H$ and then right to $L_{1}$; triangulate by 


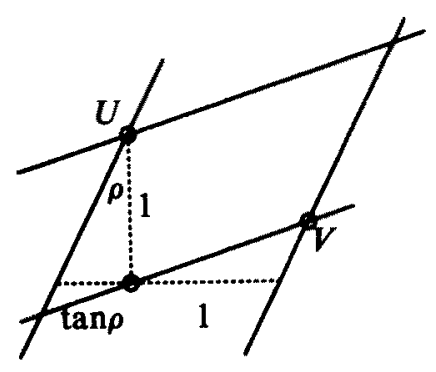

(a)

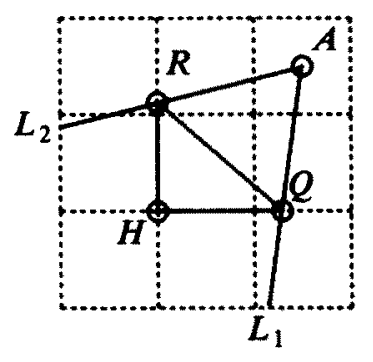

(b)

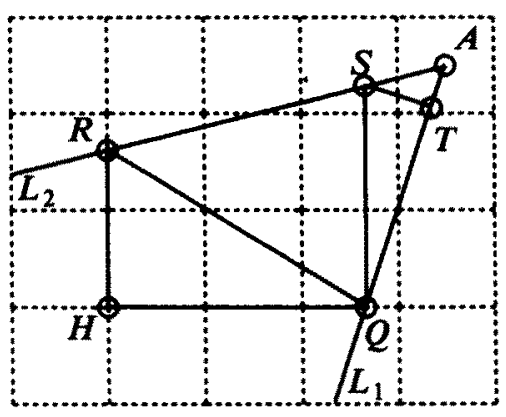

(c)

Fig. 4.7. Lemma 4.5, Case 1.

a diagonal. The lower boundary of the quadrilateral has length in [ $3-2 \tan \rho, 3-$ $\tan \rho$ ). If this length is in $[1,2)$, we are done. Otherwise, we apply Lemma 4.3 below $H Q$.

Case 2. Suppose $L_{2}$ is in octant 6 and $L_{2}$ is clockwise from $L_{1}$. Find the top horizontal gridline with exactly four gridpoints between or on $L_{1}$ and $L_{2}$. Let $M$ and $R$ be its intersection points with $L_{2}$ and $L_{1}$, respectively, as shown in Fig. 4.8. Place a vertex $P$ at the neighbor point of $M$. Then $|P R| \in[2,3)$. Extend a perpendicular from $P$, intersecting $L_{2}$ at $U$. Apply Lemma 4.3 below $P R$ to obtain a satisfactory boundary. If $\angle A U R$ and $\angle A R U$ are nonobtuse, we are done. Otherwise, draw a perpendicular as shown in Fig. 4.8.

Case 3. Suppose $L_{2}$ is in octant 7. Let $\sigma, 0 \leq \sigma \leq 45^{\circ}$, be the angle of $L_{2}$ with the vertical. Find the highest horizontal gridline such that either four or five points lie between or on $L_{1}$ and $L_{2}$. Let $U$ and $V$ be where the gridline one unit higher intersects $L_{1}$ and $L_{2}$, respectively, as shown in Fig. 4.9. $|U V|<4$. The slopes of $L_{1}$ and $L_{2}$ guarantee that $|U V| \geq 1$.

We claim there is a point $C$ on $U V$ such that $C$ lies above $P Q,|U C| \leq 2$, $|C V| \leq 2$, and $|U C| \in[|U V| / 3,2|U V| / 3]$. If the halfway point between $U$ and $V$ lies above $P Q$, take $C$ to be this halfway point. Otherwise, suppose without loss of generality that the halfway point lies too far to the right to be above $P Q$. 


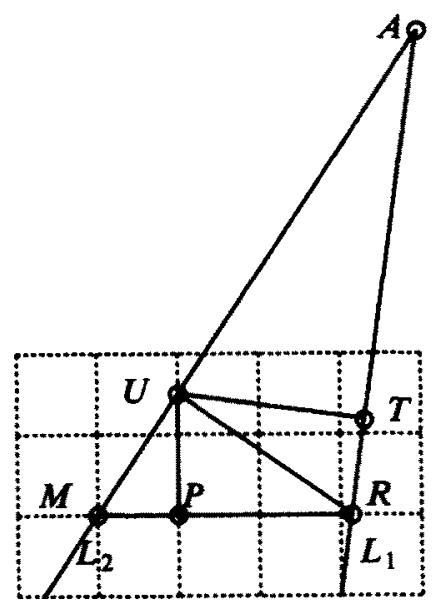

Fig. 4.8. Lemma 4.5, Case 2.

Take $C$ to be the gridpoint one unit above $Q$. Since $|Q R| \leq 2,|C V| \leq 2$ as well. Also, $|U C|<|U V| / 2 \leq 2$. Let $x_{1}=|M P|$ and $x_{2}=|Q R|$. Note that $|P Q|=1$, or the halfway point between $U$ and $V$ would have been above $P Q$. Since $x_{1} \geq 1$, $\tan \rho \leq 1$, and $x_{2} \leq 2$, we have

$$
|U C|-|U V| / 3=\left(x_{1}+1-\tan \rho\right)-\left(x_{1}+x_{2}+1-\tan \rho-\tan \sigma\right) / 3 \geq 0 .
$$

Triangulate the region $M R V C U$ as shown in Fig. 4.9. To triangulate above $U C V$, let $t=\tan \sigma+\tan \rho$ and $z=|U V|$. Let $H J$ be a horizontal line connecting $L_{1}$ and $L_{2}$ at height $y$ above $U V$, where (a) if $t \geq 1$, then $y=z / 3$, and (b) if $t<1$, then $y=z /(2+t)$. Triangulate as shown in Fig. 4.9.

Case 4. Suppose $L_{2}$ is in octant 8 . Let $\sigma$ be the angle of $L_{2}$ with the horizontal, $0 \leq \sigma \leq 45^{\circ}$. We consider two subcases, according to whether $\rho \leq \tan ^{-1}\left(\frac{2}{5}\right)$.

First, suppose $\rho \leq \tan ^{-1}\left(\frac{2}{5}\right)$. We begin by showing the existence of a neighbor gridpoint $Y$ of $L_{2}$ whose horizontal distance to $L_{1}$ is in the range $[2,4)$ (see Fig. 4.10). There is a vertical gridline $g$ that lies at least 2 units and less than 3 units

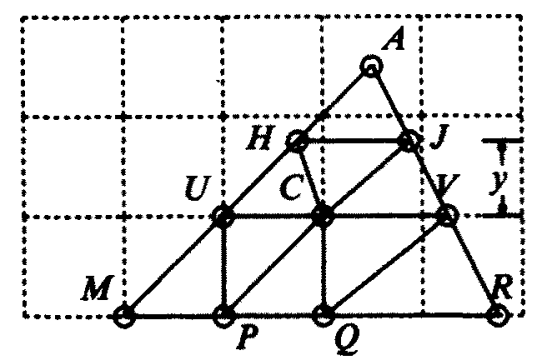

Fig. 4.9. Triangulation for Lemma 4.5, Case 3. 


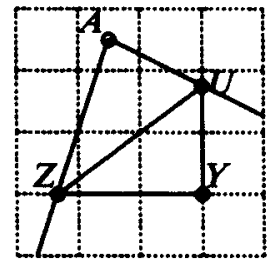

Fig. 4.10. Triangulation for Lemma 4.5, Case 4, when $\rho \leq \tan ^{-1}\left(\frac{2}{5}\right)$.

to the right of $A$. Since $\sigma \leq 45^{\circ}, L_{2}$ drops less than 3 units before intersecting $g$. Let $G$ be the neighbor point of the intersection of $g$ with $L_{2}$. Then $A_{y}-G_{y}<5$. The horizontal distance from $G$ to $L_{1}$ is in the range $[2,5)$. If this distance is less than 4, we are done, with $Y=G$. Otherwise, let $G_{1}$ be the gridpoint one unit to the left of $G$. The horizontal distance from $G_{1}$ to $L_{1}$ is in $[3,4)$. The veritcal distance to $L_{2}$ is in the range $[1,3)$. If this distance is less than 2 , we are done, with $Y=G_{1}$. Otherwise, let $Y$ be one unit above $G_{1}$. The vertical distance from $Y$ to $L_{2}$ is now in $[1,2)$. The horizontal distance from $Y$ to $L_{1}$ is in $[13 / 5,4)$, and we are done.

Let $U$ be the point of $L_{2}$ above $Y$, and $Z$ the point on $L_{1}$ to the left of $Y$. Triangulate as shown in Fig. 4.10. Finally, to obtain a satisfactory boundary, trianguate below $Z Y$ as in Lemma 4.4.

Now, suppose $\rho>\tan ^{-1}\left(\frac{2}{5}\right)$. Consider the points at which two successive vertical gridlines intersect $L_{2}$, and the horizontal lines through these intersection points from $L_{2}$ to $L_{1}$, as shown in Fig. 4.11(a). The difference in length of the horizontal lines is at most 2 since $\sigma, \rho \leq 45^{\circ}$. Hence, there exists a vertical gridline for which the horizontal segment $W U$ has a length in the range $[1,3)$, as shown in Fig. 4.11(b). Define $x_{1}=|W U|$. Let $Y$ be the gridpoint below $U$ by a distance in $[1.5,2.5)$. Let $Z$ be on $L_{1}$ to the left of $Y$, and let $x_{2}=|Y Z|$. Since $\frac{2}{5}<\tan \rho \leq 1$, $x_{2} \in[1.6,5.5)$. If $Y$ is the neighbor point of $U, U Y$ is a satisfactory boundary edge. Otherwise, triangulate to the right of $U Y$ as in Lemma 4.3 (rotated). Next,

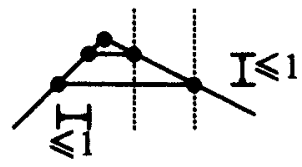

(a)

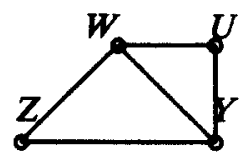

(c)

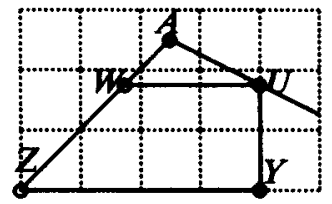

(b)

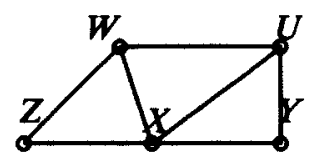

(d)

Fig. 4.11 Triangulation for Lemma 4.5, Case 4, when $\rho>\tan ^{-1}\left(\frac{2}{5}\right)$. 
we show how to triangulate the quadrilateral $W U Y Z$, and how to obtain a satisfactory boundary below WUYZ.

If $x_{2} \in[1.6,3)$, triangulate by drawing $W Y$. If $x_{2} \in[1.6,2)$, then $Z Y$ gives a good interface. If $x_{2} \in[2,3)$, triangulate below $Z Y$ by Lemma 4.4. If $x_{2} \in[3,5.5)$, let $X$ be the gridpoint that lies $\left\lfloor x_{1}\right\rfloor$ to the left of $Y$; triangulate as shown in Fig. 4.11(b). To see that $\angle W X U$ is at least $\tan ^{-1}\left(\frac{1}{4}\right)$, draw a perpendicular from $X$ to $W U$. Either one segment of $W U$ is at least 0.625 and one subangle is at least $\tan ^{-1}\left(\frac{1}{4}\right)$, or both segments are at least 0.375 and both subangles are at least $\tan ^{-1}(0.375 / 2.5)>0.5 \tan ^{-1}\left(\frac{1}{4}\right)$. A similar argument applies to $\angle Z W X$.

From above, $1 \leq|Z X|<y+1 \leq 3.5$. If $|Z X|<2$, then $Z X$ is a satisfactory boundary edge. Otherwise, Lemma 4.4 is applied below $Z X$ to obtain a satisfactory boundary.

With rotations and reflections the following three lemmas can be combined to triangulate near any obtuse or reflex angle. Choose a local origin so that $A$ is in the cell with lower left corner at $(0,0)$.

Lemma 4.6. Let $U=(2,-1), V=(3,-1), W=(3,1), X=(3,2)$ and $Y=(2,2)$ as in Fig. 4.12. Then the triangulation shown of region AUVWXY is good.

Proof. Straightforward.

Lemma 4.7. Let $B A C$ be an obtuse angle such that $A B$ is in the second octant at $A$.

(a) Suppose the edge $A C$ is in the seventh octant at $A$. Let $E$ be the point of intersection of $A B$ and the line $y=2, F$ be the point of intersection of $A C$ and $y=-1, U=(5,-1)$, and $V=(5,2)$. Then the region $A F U V E$ can be well-triangulated, with new points introduced as necessary to make the boundary EVUF satisfactory (Fig. 4.13).

(b) Suppose the edge $A C$ is in the eighth octant at $A$. Let $W$ be the point of intersection of $A B$ and the line $y=3$, and let $F$ be the point of intersection of $A C$ and the line $x=7$, and let $W=(7,3)$. Then the region $A F W E$ can be well-triangulated, with new points introduced as necessary to make the boundary FWE satisfactory (Fig. 4.14).

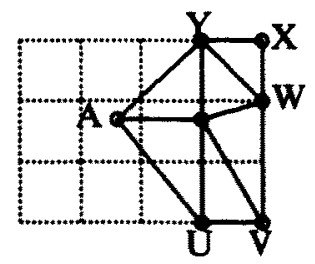

Fig. 4.12. Triangulation for Lemma 4.6. 


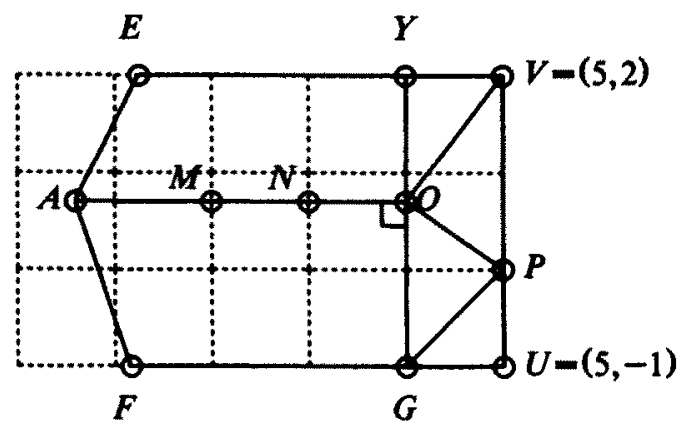

(a) Subregions

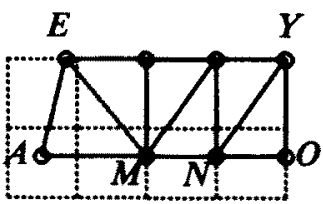

(b) $E_{\mathrm{x}} \leq 1$

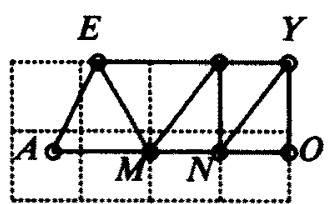

(c) $1<E_{\mathrm{r}} \leq 2$

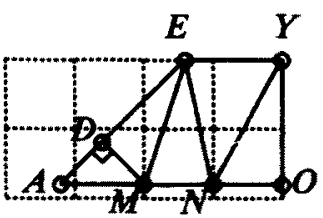

(d) $2<E_{x} \leq 3$

Fig. 4.13. Case (a) of Lemma 4.7.

Proof. Most angles can be shown to be between $\tan ^{-1}\left(\frac{1}{4}\right)$ and $90^{\circ}$ by either the semicircle principle, inspection based on given constraints of position and slopes, or the inequality $\tan \left(\alpha_{1}+\alpha_{2}\right) \geq \tan \left(\alpha_{1}\right)+\tan \left(\alpha_{2}\right)$, usually applied by dropping a perpendicular from a point to the opposite side of the triangle. The triangulations are suggested by the Figs. 4.15-4.17.

The division of Lemma 4.7 into Cases (a) and (b) is induced by the satisfactory path requirement of an edge nearly perpendicular to $A C$.

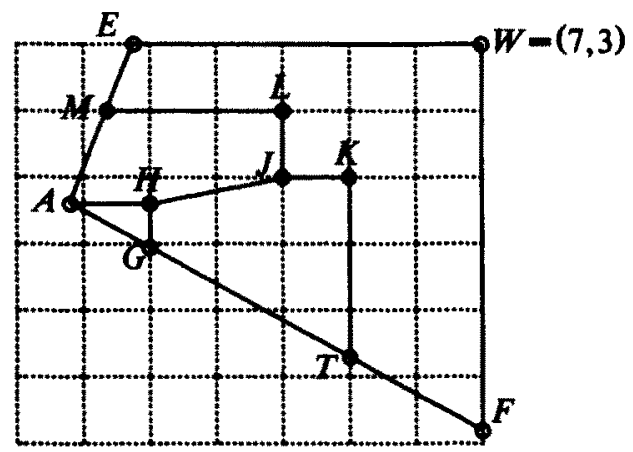

Fig. 4.14. Lemma 4.7, Case (b), when the slope of $A F$ is less than or equal to $-\frac{1}{2}$. 


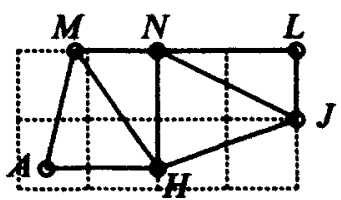

(a) $M_{x} \leq 1$

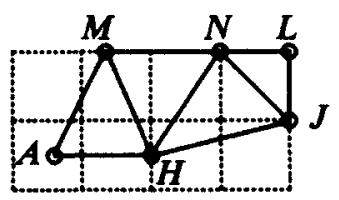

(b) $1<M_{x} \leq 2$

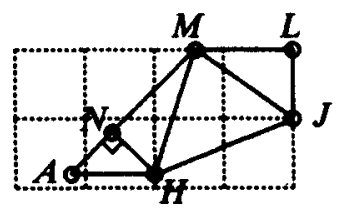

(c) $2<M_{x} \leq 3$

Fig. 4.15. Triangulations for $A H J L M$ of Fig. 4.14.
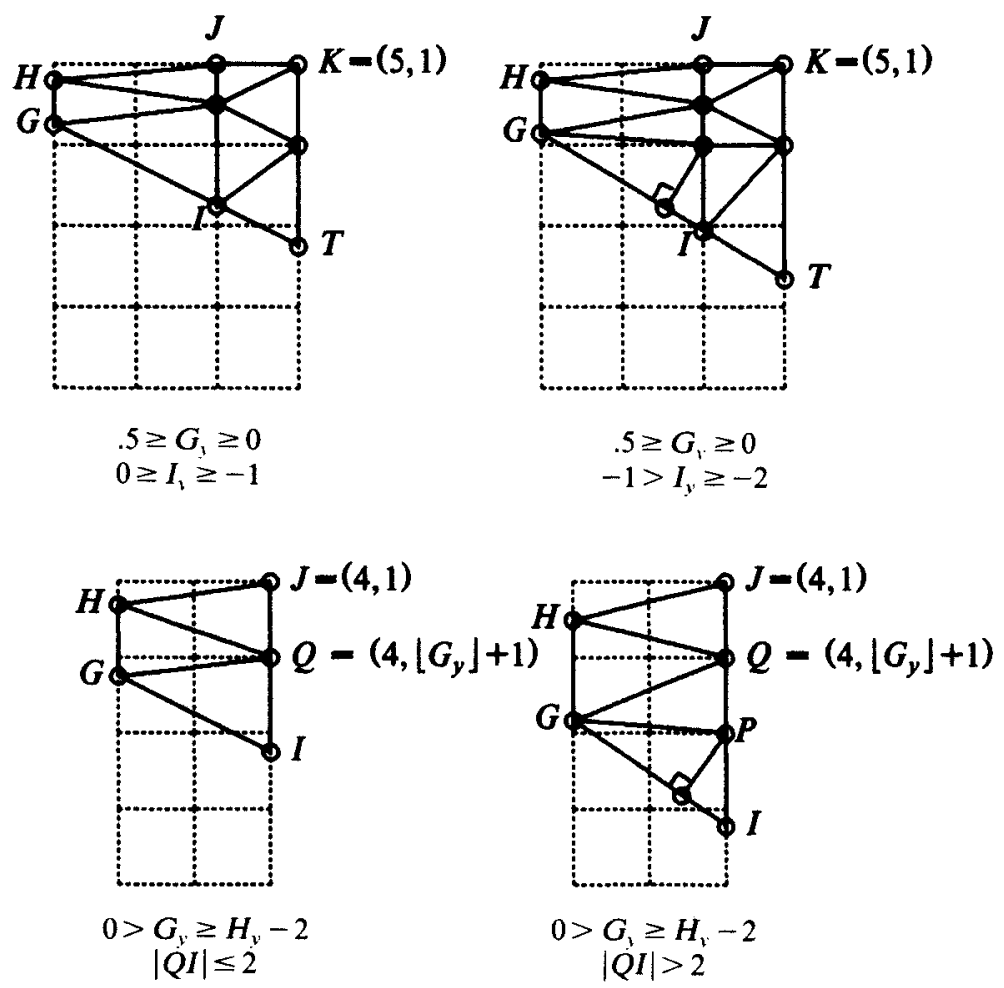

Fig. 4.16. Further divisions of Fig. 4.14.

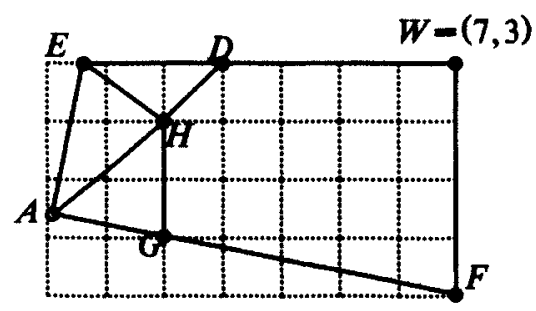

(a)

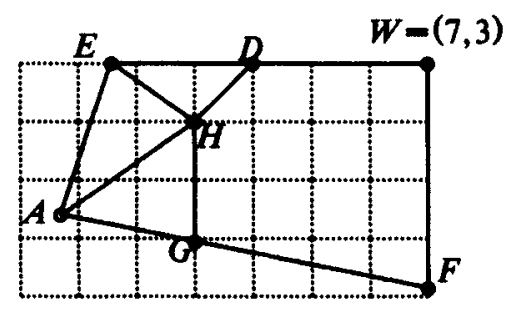

(b)

Fig. 4.17. Lemma 4.7, Case (b), when the slope of $A C$ is greater than $-\frac{1}{2}$. 


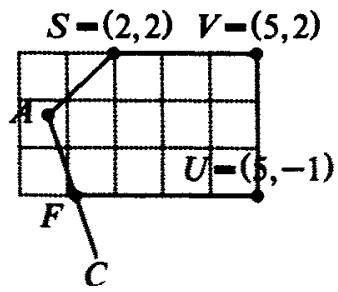

(a)

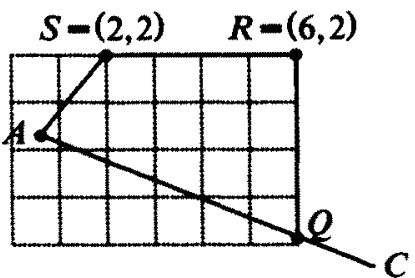

(b)

Fig. 4.18. Triangulatable regions for Lemma 4.8 .

Lemma 4.8. Let $S=(2,2)$ and let $A C$ be in the seventh or eighth octant of $A$.

(a) Suppose $A C$ is in the seventh octant of $A$, as shown in Fig. 4.18(a). Let $F$ be the point of intersection of $A C$ and the line $y=-1, U=(5,-1)$, and $V=(5,2)$. Then AFUVS can be well-triangulated, with new points to make sides $F U, U V$, and VS satisfactory, and with no points introduced on $A S$.

(b) Suppose $A C$ is in the eighth octant of $A$, as shown in Fig. 4.18(b). Let $R=(6,2)$, and let $Q$ be the point of intersection of $A C$ and $x=6$. Then $A Q R S$ can be well-triangulated, with new points introduced to make sides $Q R$ and $R S$ satisfactory, and with no points introduced on AS.

Proof. (a) $A C$ in the seventh octant, i.e., nearly vertical with negative slope. In this case, apply the same triangulation as in Case (a) of Lemma 4.7 , i.e. with $E=S=(2,2)$ in Fig. 4.13(a). Thus, Fig. 4.13(c) is applied for the region $A O Y E$ of Fig. 4.13(a). This method well-triangulates the region even if the slope of $A S$ is nearly horizontal, instead of nearly vertical as specified in Lemma 4.7.

(b) $A C$ in the eighth octant, i.e., nearly horizontal with negative slope. First, suppose $S A F$ is obtuse. Then $A S$ must lie in the second octant at $A$, but the position of $S$ forces the slope of $A S$ to be at most 2 . Hence, the slope of $A C$ is at most $-\frac{1}{2}$. We can apply the triangulation method used in Case (b) of Lemma 4.7 when the slope of $A C$ is at most $-\frac{1}{2}$ (ignoring the rectangle $M L V W E$ of Fig. 4.14 since it lies outside the desired region for Lemma 4.8).

Now, suppose $S A F$ is acute. The region to be triangulated is shown in Fig. 4.18(b). Draw a vertical line downward from $S$, intersecting $A C$ at $P$. If $|S P| \geq 2$, apply Lemma 4.4 (rotated) to triangulate next to $S P$. Now, either all of $S P R W$ is well-triangulated, or all but the rightmost 2 or 4 units is well-triangulated. In the latter case, make $R Q$ satisfactory and triangulate the remaining region by Lemma 4.2 .

Lemma 4.9. Let $A$ be the vertex of an obtuse or reflex angle of $R$. Then there is a region around $A$ that can be well-triangulated with a satisfactory path along its boundary.

Proof. Without loss of generality we may assume $A B$ lies in the first quadrant of $A$ and bounds the interior of $P$ from above. We consider cases according to the quadrant of $A C$, as in Fig. 4.19. 


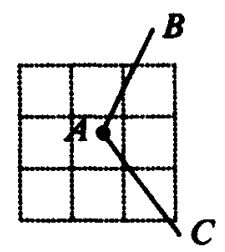

(a)

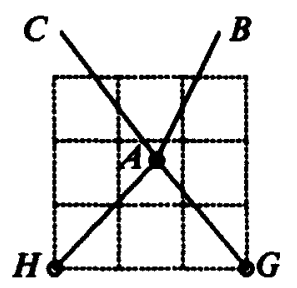

(c)

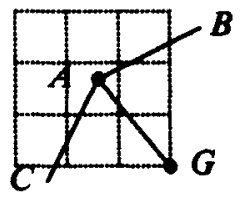

(b)

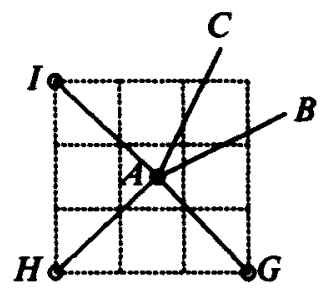

(d)

Fig. 4.19. Cases for triangulating at $\boldsymbol{A}$.

(a) Apply Lemma 4.7, either directly or reflected.

(b) Apply Lemma 4.8 to $C A G$, and a reflection of Lemma 4.8 to $B A G$. Note that the two regions obtained do not overlap except along the boundary edge $A G$, and no points are added on $A G$ by Lemma 4.8 or its reflection.

(c) Apply Lemma 4.8 to angles $B A G$ and $C A H$ and Lemma 4.6 to $H A G$.

(d) Apply Lemma 4.8 to angles $B A G$ and $C A I$, and Lemma 4.6 to $H A G$ and HAI.

When the above triangulations are applied at the vertices of $P$, the remaining region satisfies conditions (1)-(3) as desired.

Combining Lemmas $4.2,4.5$, and 4.8 , we obtain the main result.

Theorem 4.10. Any polygon can be triangulated using no obtuse angles and no angles smaller than $\tan ^{-1}\left(\frac{1}{4}\right)$ or the minimum angle of the polygon, whichever is smaller.

\section{Concluding Remarks}

Our algorithms demonstrate that a polygon can be triangulated without obtuse angles. But the topic is by no means exhausted, because there are many combinations of side conditions that could be imposed resulting in simpler (or more complicated) algorithms. For example, Lynn Wilson has devised a much simpler scheme that suffices for certain interface problems [19]. A hexagonal grid might be investigated. It would be interesting to see if the independent cell triangulations given here can be used to repair locally obtuse triangulations given by other algorithms. 


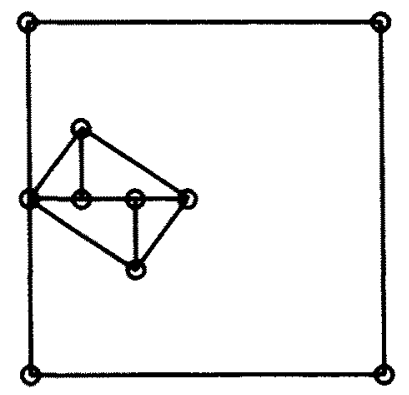

Fig. 5.1. Two sides of a crack are independent.

We defined our problem in terms of a simple polygon. In fact, it is sufficient to assume that $P$ is the closure of a bounded planar open set whose boundary is composed of finitely many straight line segments. We consider a "crack" to be made up of two line segments and allow different points on the two segments. In effect, we perturb the problem to open the crack into an infinitesimally narrow wedge (Fig. 5.1).

Let $n$ be the number of cells along the boundary. (Recall that the grid spacing depends on the polygon vertex angles and the separation of nonadjacent polygon edges.) Each cell is partitioned into a bounded number of triangles. With coarse grading of the interior as indicated in Fig. 2.2, there will be $O(n \log n)$ triangles inside; otherwise there would be $O\left(n^{2}\right)$. But in either case the pattern is so regular that little processing is needed for the interior. Thus the number of triangles and the runtime of the algorithm is for practical purposes linear in the gridsize neeeded to resolve the polygon. At least this many triangles would ordinarily be desired in the finite element method to provide an adequate approximating space.

\section{Acknowledgments}

We thank Randy Bank, Mark Pinto, and Don Rose for discussions of the finite element context and the referee for pointing out [8].

\section{References}

1. I. Babuska and A. K. Aziz, On the angle condition in the finite element method, SIAM J. Numer. Analy. 13 (1976), 214-226.

2. B. Baker, E. Grosse, and C. Rafferty, Non-obtuse triangulation of polygons, Numerical Analysis Manuscript 84-4, AT\&T Bell Laboratories, Murray Hill, NJ, 1985.

3. R. E. Bank, PLTMG User's Guide, 1985.

4. R. E. Bank, D. J. Rose, and W. Fichtner, Numerical methods for semiconductor device simulation, IEEE Trans. Electron Devices 30 (1983), 1031-1041.

5. B. Chazelle and J. Incerpi, Triangulation and shape-complexity, Report CS $83-22$, Department of Computer Science, Brown University, 1983.

6. 1. Fried, Condition of finite element matrices generated from nonuniform meshes, AIAAJ.10 (1972), 219-221. 
7. M. R. Garey, D. S. Johnson, F. R. Preparata, and R. E. Tarjan, Triangulating a simple polygon, Inform. Process. Lett. 74 (1978), 4.

8. J. L. Gerver, The dissection of a polygon into nearly equilateral triangles, Geom. Dedicata 16 (1984), 93-106.

9. P. J. Green and R. Sibson, Computing Dirichlet tessellations in the plane, Comput. J. 21 (1978), 168-173.

10. S. E. Laux and B. M. Grossman, A general control-volume formulation for modeling impact ionization in semiconductor transport, IEEE Trans. Electron Devices 32 (1985), 2076-2081.

11. C. L. Lawson, Generation of a triangular grid with application to contour plotting, Technical Memorandum 299, California Institute of Technology, JPL, 1972.

12. R. H. MacNeal, An asymmetrical finite difference network, Quart. Appl. Math. 11 (1953), 295-310.

13. R. R. O'Brien et al., Two-dimensional process modeling, IBM J. Res. Develop. 29 (1985), 229-241.

14. H. Samet, The quadtree and related hierarchical data structures, ACM Comput. Surveys 16 (1984), $187-260$.

15. M. S. Shephard and M. A. Yerry, An approach to automatic finite element mesh generation, Comput. Engrg 3 (1982), 21-27.

16. R. Sibson, Locally equiangular triangulations, Comput. J. 21 (1978), 243-245.

17. G. Strang and G. J. Fix, An Analysis of the Finite Element Method, Prentice-Hall, Englewood Cliffs, NJ, 1973.

18. R. S. Varga, Matrix Iterative Analysis, Prentice-Hall, Englewood Cliffs, NJ, 1962.

19. L. O. Wilson, A non-obtuse triangulation which follows a moving interface, Technical Memorandum, AT\&T Bell Laboratories, Murray Hill, NJ, 1985.

Received December 13, 1985, and in revised form December 1, 1986. 